Research, Society and Development, v. 9, n. 11, e3759119865, 2020

(CC BY 4.0) | ISSN 2525-3409 | DOI: http://dx.doi.org/10.33448/rsd-v9i11.9865

\title{
Dislipidemia na infância e adolescência: um olhar para educação em saúde
}

Dyslipidemia in childhood and adolescence: a view at health education

Dislipidemia en la infancia y la adolescencia: una mirada a la educación para la salud

Recebido: 02/11/2020 | Revisado: 08/11/2020 | Aceito: 14/11/2020 | Publicado: 18/11/2020

\section{Susana Nogueira Diniz}

ORCID: https://orcid.org/0000-0002-4329-848X

Universidade Anhanguera de São Paulo, Brasil

E-mail: susana.diniz@anhanguera.com

Thiago da Silva Lima

ORCID: https://orcid.org/0000-0001-9112-2553

Universidade Anhanguera de São Paulo, Brasil

E-mail: thiagosilvaupexpress@ hotmail.com

Audrey de Souza Marques

ORCID: https://orcid.org/0000-0001-7097-4204

Universidade Anhanguera de São Paulo, Brasil

Universidade do Norte do Paraná, Brasil

E-mail: audrey.marquez@ unopar.br

Cristina Eunice Okuyama

ORCID: https://orcid.org/0000-0003-0876-4331

Universidade Anhanguera de São Paulo, Brasil

E-mail: cristina.e.costa@anhanguera.com

\section{Resumo}

Este estudo teve como objetivo realizar um diagnóstico situacional da prevalência de dislipidemias em crianças e adolescentes no Brasil. E, verificar na Base Nacional Comum Curricular (BNCC) se há uma proposta de abordagem nas escolas brasileiras sobre hábitos saudáveis de vida e promoção da saúde. Trata-se de pesquisa qualitativa e quantitativa utilizando-se da abordagem da revisão integrativa de dados do perfil lipídico disponíveis em teses e dissertações. A avaliação interpretativa da BNCC foi realizada através da descrição de habilidades e competências das disciplinas de Educação Física, Ciências e Biologia para verificar a presença do tema promoção da saúde e provocar uma reflexão sobre maneiras ou 
formas de abordagem desse conteúdo no ensino do país. Os resultados demonstram que é alta a prevalência de dislipidemia em crianças e adolescentes propiciando um potencial risco para o desenvolvimento de doença cardiovascular na vida adulta. Além disso, de acordo com a BNCC, as diretrizes de orientação do tema nos âmbitos escolares ocorrem de forma discreta e somente após a idade na qual os hábitos individuais já estão estabelecidos. Portanto, este estudo mostra a necessidade de medidas para o diagnóstico precoce, associado a programas de prevenção das dislipidemias e ações de educação, conscientização e definição de políticas públicas voltadas à promoção da saúde. Além disso, sugere que o conhecimento científico articulado ao Ensino de Ciências, pode estimular o processo de percepção dos educandos sobre a importância de uma rotina de vida fisicamente ativa e a implantação de hábitos alimentares saudáveis, para a promoção da saúde.

Palavras-chave: Adolescente; Criança; Dislipidemia; Fator de risco; Saúde pública; Ensino.

\begin{abstract}
This study aimed to make a diagnosis of the prevalence of dyslipidemia in children and adolescents in Brazil and check in the Base Nacional Comum Curricular (BNCC) if there is a suggestion to approach Brazilian schools on healthy living habits and health promotion. This research used a qualitative and quantitative approach of integrative review on lipid profile data available in theses and dissertations. The BNCC interpretative assessment was carried out by describing the skills and competences of the disciplines of Physical Education, Science and Biology to verify the presence of the theme health promotion and incite a reflection on ways of approaching this content in the country's education system. The results demonstrate that the prevalence of dyslipidemia in children and adolescents is high, providing a potential risk for the development of cardiovascular disease in adulthood. In addition, the guidelines for guiding the topic in school settings occur discreetly and only after the age at which individual habits are already established. Therefore, this study shows the need for measures of early diagnosis, associated with programs for the prevention of dyslipidemia, and actions for education, awareness and the definition of public policies aimed at health promotion. And it suggests that scientific knowledge, linked to Science Education, can stimulate the students' perception process about the importance of a physically active life routine and healthy eating habits, for health promotion.
\end{abstract}

Keywords: Adolescent; Child; Dyslipidemia; Risk factor; Public health; Teaching. 


\section{Resumen}

Este estudio tuvo como objetivo realizar un diagnóstico de la prevalencia de dislipidemia em niños y adolescentes em Brasil y examinar em la Base Nacional Comum Curricular (BNCC) si hay un enfoque sobre hábitos de vida saludables y promoción de la salud em las escuelas brasileñas. Se trata de una investigación cualitativa, cuantitativa y integradora de los catos del perfil lipídico disponibles em tesis y disertaciones. La evaluación interpretativa del BNCC se realizó por el análisis de las habilidades y competencias de las disciplinas de Educación Física, Ciencia y Biología para verificar la existencia del tema de promoción de la salud y provocar una reflexión sobre las formas de abordar este contenido em la enseñanza del país. Los resultados demuestran que la prevalencia de dislipidemia em niños y adolescentes es alta, lo que genera un riesgo potencial para el desarrollo de enfermedad cardiovascular em la edad adulta. Además, según el BNCC, las pautas para orientar la temática en el ámbito escolar se dan de forma discreta y solo después de la edad em la que los hábitos individuales ya están establecidos. Este estudio muestra la necesidad de medidas de diagnóstico precoz asociadas a programas de prevención de dislipidemias y acciones de educación, sensibilización y definición de políticas públicas orientadas a la promoción de la salud. Igualmente, sugiere la Enseñanza de las Ciencias puede estimular el proceso de percepción de los estudiantes sobre la importancia de una rutina de vida física activa y alimentaciones saludables, para la promoción de la salud.

Palabras clave: Adolescente; Niño; Dislipidemia; Factor de riesgo; Salud pública; Enseñanza.

\section{Introdução}

A concepção desta pesquisa se deu a partir de questionamentos que surgiram de um profissional da área de Educação Física que atua na rede pública de ensino há 7 anos. Durante este período e observando a postura dos alunos se alimentando e cada vez mais sedentários, surgiram alguns incômodos, relacionados principalmente a alimentação e atividade física na escola. A partir deste momento, começaram a reflexão sobre a importância de se discutir esses temas com os alunos. Muitos estudantes costumam ignorar os alimentos ofertados pela escola, pois preferem os alimentos que eles mesmos costumam chamar de "mais gostosos", como coxinha, salgadinhos, biscoito, pirulito, bala e afins, esses alimentos aumentam muito os níveis de colesterol caracterizando um importante fator de risco para doenças cardiovasculares. Outro fato também observado a cada ano que passa, é que nossas crianças 
vêm utilizando o chamado "lazer sedentário", através de jogos eletrônicos criando assim uma possibilidade muito grande de serem adultos sedentários e com uma alimentação de má qualidade.

Diante do exposto fica evidente a necessidade do incentivo da manutenção de uma rotina de vida fisicamente ativa e a implantação de hábitos alimentares saudáveis na infância que podem ser importantes medidas para a promoção da saúde e para o controle do colesterol na população. O Paradigma do estilo de vida mostra que o homem contemporâneo utiliza cada vez menos suas potencialidades e, que a baixa utilização da atividade física pode contribuir de forma significativa para o desenvolvimento de diversas doenças crônicas não transmissíveis. Identifica-se assim, neste paradigma, a necessidade da incorporação da prática de atividade física para reduzir essa problemática. Visando assim, a interação das dimensões da promoção da saúde, da qualidade de vida e da atividade física dentro de um movimento denominado “Movimento Vida Ativa”. Diversos estudos reiteram a associação entre a prática de atividade física regular e a melhoria da qualidade de vida do indivíduo (Campos et al., 2019; Guedes \& Guedes, 1995; Matsudo et al., 2012; Silva et al., 2010). Um exemplo atual, de que a rotina sedentária favorece a um aumento no ganho de peso corporal e surgimento de comorbidades associadas ao maior risco cardiovascular, foi a recomendação de isolamento social impostas em vários países devido à rápida e incontrolável disseminação do novo coronavírus (SARS-CoV-2) (Ferreira et al., 2020).

Já se passaram mais de 30 anos que Martins et al (1989) levantaram a hipótese de que os distúrbios lipídicos podiam constituir-se em grave problema de Saúde Pública no Brasil. As dislipidemias são distúrbios lipídicos caracterizados pela alteração do metabolismo de lipoproteínas circulantes no sangue. No Brasil, existem diretrizes informativas sobre as dislipidemias estabelecidas pelo Departamento de Aterosclerose da Sociedade Brasileira de Cardiologia (SBC-DA) juntamente com a Sociedade Brasileira de Diabetes e a Sociedade Brasileira de Endocrinologia e Metabologia (SBEM). As dislipidemias podem ser classificadas em hiperlipidemia e hipolipidemia. As hiperlipidemias são definidas pela elevação de colesterol e/ou de triglicerídeos no plasma, enquanto as hipolipidemias são indicadas pelos baixos níveis de HDL. Todas essas alterações contribuem com as disfunções endoteliais, levando ao desenvolvimento da aterosclerose (Shattat, 2014). Na prática clínica, as hiperlipidemias são as dislipidemias mais relevantes devido sua frequência (Castro Cabezas et al., 2018).

A dislipidemia que surge de forma silenciosa na infância e representa um risco à saúde na fase adulta está muitas vezes associada ao consumo alimentar e estilo de vida do indivíduo 
(Turer et al., 2018). Segundo a I Diretriz de Prevenção da Aterosclerose na Infância e na Adolescência de 2005 é necessário promover intervenções profiláticas para uma formação da personalidade com um estilo de vida mais saudável, destacando a importância da inserção de práticas relacionadas à nutrição e atividade física na prevenção e tratamento de doenças cardiovasculares nas crianças. As intervenções aplicadas na infância e adolescência aumentam as chances de sucesso na sedimentação dos hábitos de vida à medida que são repetidos (Bays et al., 2016; Brown, 2018). Com isso, a iniciativa de educação em saúde, para adoção de modos de vida saudáveis, poderá servir para fortalecer ações de promoção da saúde junto aos escolares em relação à alimentação saudável, assim como, a orientação da importância da educação alimentar e da prática de atividade física.

Acreditamos que uma forma de abranger e alcançar esse público-alvo poderia ser preconizada pela Base Nacional Curricular Comum (BNCC), que é um documento normativo e progressivo de aprendizagens essenciais para alunos desenvolverem durante toda a Educação Básica no Brasil. Este documento propõe para as diferentes esferas de ensino o que poderia ser abordado com os educandos ao longo das diferentes etapas escolares, em função das características de cada região, observando a importância de se considerar as características da comunidade e os conhecimentos prévios dos alunos sem esquecer de desenvolver os conceitos e noções mínimos exigidos para preparar o estudante para a vida escolar e profissional, sem prejuízo para menos favorecidos, dando assim a mesma oportunidade para todos (Brasil, 2017).

Assim, configura-se então, o problema da pesquisa: Será que as escolas, e a comunidade estão conscientes para educar e prevenir os fatores de risco para doenças cardiovasculares? Como abordar aspectos da alimentação saudável nas escolas? Qual a importância da atividade física na prevenção desses fatores de risco? Será que essas temáticas são abordadas na Base Nacional Comum Curricular (BNCC). Desta forma, este estudo tem como principal objetivo realizar um levantamento das dislipidemias, que são os principais fatores de risco para doenças cardiovasculares em crianças e adolescentes, e verificar na BNCC a existência da abordagem de hábitos saudáveis de vida para promoção da saúde, nas escolas brasileiras. 


\section{Metodologia}

\subsection{Revisão integrativa da literatura}

Trata-se de uma revisão integrativa da literatura, visto que esse tipo de pesquisa "é a mais ampla abordagem metodológica referente às revisões, permitindo a inclusão de estudos experimentais e não-experimentais para uma compreensão completa do fenômeno analisado" (Souza et al., 2010, p.103). Esta revisão permite responder perguntas específicas através de métodos sistemáticos que auxiliam na identificação, seleção e avaliação dos estudos que serão incluídos no trabalho. É um método específico, que apresenta o estado da arte sobre um tema contribuindo para o desenvolvimento de teorias e para fornecer uma compreensão mais abrangente de um fenômeno particular (Mendes et al., 2008). Esse método de pesquisa objetiva traçar uma análise sobre o conhecimento já construído em pesquisas anteriores sobre um determinado tema. Possibilita a síntese de vários estudos já publicados, permitindo a geração de novos conhecimentos, pautados nos resultados apresentados pelas pesquisas anteriores (Benefield, 2003; Mendes et al., 2008; Polit \& Beck, 2006).

Segundo Redeker (2000) a revisão integrativa é particularmente útil para "um sumário da literatura, num conceito específico ou numa área de conteúdo, em que a pesquisa é sumariada (resumida), analisada, e as conclusões totais são extraídas". A construção da presente revisão integrativa baseia-se principalmente nos estudos de Cooper (1984), Ganong (1987), Mendes (Mendes et al., 2008), Beyea \& Nicoll (1998), Stetler et al. (1998) e Whitemore (2005).

Os descritores ou palavras-chave, da estratégia de busca, definidos neste estudo foram: “dislipidemias, criança, adolescente, fator de risco e saúde pública" e os bancos de dados utilizados foram: "base de dados CAPES Teses e de busca manual na internet através do Google". Neste estudo levou-se em consideração que a maioria dos artigos científicos se baseiam em pesquisas acadêmicas de Mestrado e Doutorado, esta revisão passou a se restringir a análise de dissertações e teses.

Os trabalhos foram selecionados somente na língua portuguesa, aplicando-se apenas o descritor "dislipidemia" devido à grande dificuldade na filtração dos trabalhos no banco de teses da CAPES. O período avaliado foi de janeiro de 2010 a abril de 2020, com acesso ao portal CAPES Teses no dia 20/05/2020. Como não foram considerados elegíveis nenhuma das teses e dissertações que contemplavam as regiões Norte e Centro-Oeste através da busca 
no banco de teses da CAPES, foi realizada a busca manual através do Google no período de 21 a $30 / 05 / 2020$.

Os critérios de inclusão e exclusão dos trabalhos incluídos nesta pesquisa foram: "trabalhos que estavam dentro do período estipulado, encontrados no portal CAPES Teses e no Google, e que contemplavam pelo menos 2 das 4 variáveis lipídicas (CT: colesterol total; LDL-c: colesterol da lipoproteína de baixa densidade; TG: triglicérides; HDL-c: colesterol da lipoproteína de alta densidade)". Enquanto os critérios de exclusão foram: "trabalhos que apresentavam amostra com algum tipo de alteração (como doenças ou alterações préexistentes), que sofreram intervenção medicamentosa e que só tivesse valor de $\mathrm{mg} / \mathrm{dL}$ ".

Ao fim das etapas da revisão foi realizada a análise estatística descritiva e inferencial, de modo que a prevalência das dislipidemias foi obtida por comparação com os parâmetros de diretrizes preconizadas. Além disso, foi analisada a frequência de alteração dos níveis de lipídeos séricos entre os trabalhos incluídos. Os dados relativos à dislipidemia considerando os níveis de CT, HDL-c, LDL-c e TG foram tabulados e analisados. As médias da frequência de dislipidemias foram obtidas levando em consideração ao " $n$ " total dos estudos. Os dados referentes às idades foram estimados após distribuição das frequências das amostras em cada trabalho analisado. Os dados foram analisados utilizando o software GraphPadPrism 5.0.

\subsection{Análise documental interpretativa da BNCC}

A avaliação da BNCC foi realizada através da descrição de habilidades e competências das disciplinas de Educação Física, Ciências e Biologia para verificar a presença do tema promoção da saúde e promover uma reflexão sobre maneiras ou formas de abordagem desse conteúdo no ensino básico do país. Segundo Triches \& Aranda (2016), compreende-se que o documento norteador do currículo se configura como ação de uma política pública que faz parte das reformas educacionais operadas não só no Brasil, como também mundialmente, com vistas a promover melhor qualidade na educação. A definição de um currículo nacional pode facilitar o desenvolvimento do sistema educacional do País como um todo, pois o objetivo de uma Base é garantir que os alunos, independentemente da localidade, recebam a mesma oportunidade de ensino. Isso diminuiria a diferença entre os ensinos ministrados em cada estado, trazendo melhorias na qualidade de ensino (Almeida \& Júnior, 2016).

A busca de tópicos sobre risco para saúde e hábitos de vida saudável (alimentação e atividade física) foi realizada junto aos documentos oficiais da BNCC, no currículo da educação infantil ao ensino médio. 


\section{Resultados}

\subsection{Apresentação da revisão integrativa}

\subsubsection{Características das teses e dissertações incluídas}

Foram encontrados 1.401 trabalhos, acessado dia 20/05/2020 no banco de teses da Capes e no período de 21 a 30/05/2020 na busca manual no Google. Seguindo os critérios de inclusão desta revisão, 43 trabalhos foram selecionados. Destes, 21 trabalhos foram obtidos para leitura completa, e foram excluídos 4 trabalhos que apresentavam valores apenas em mg/dL das variáveis (CT, TG, LDL-c e HDL-c). Assim, na análise principal dos dados do presente estudo, somente 17 estudos foram incluídos na análise qualitativa e quantitativa. $\mathrm{O}$ fluxograma do estudo está demonstrado na Figura 1.

Figura 1 - Fluxograma da seleção das teses e dissertações.
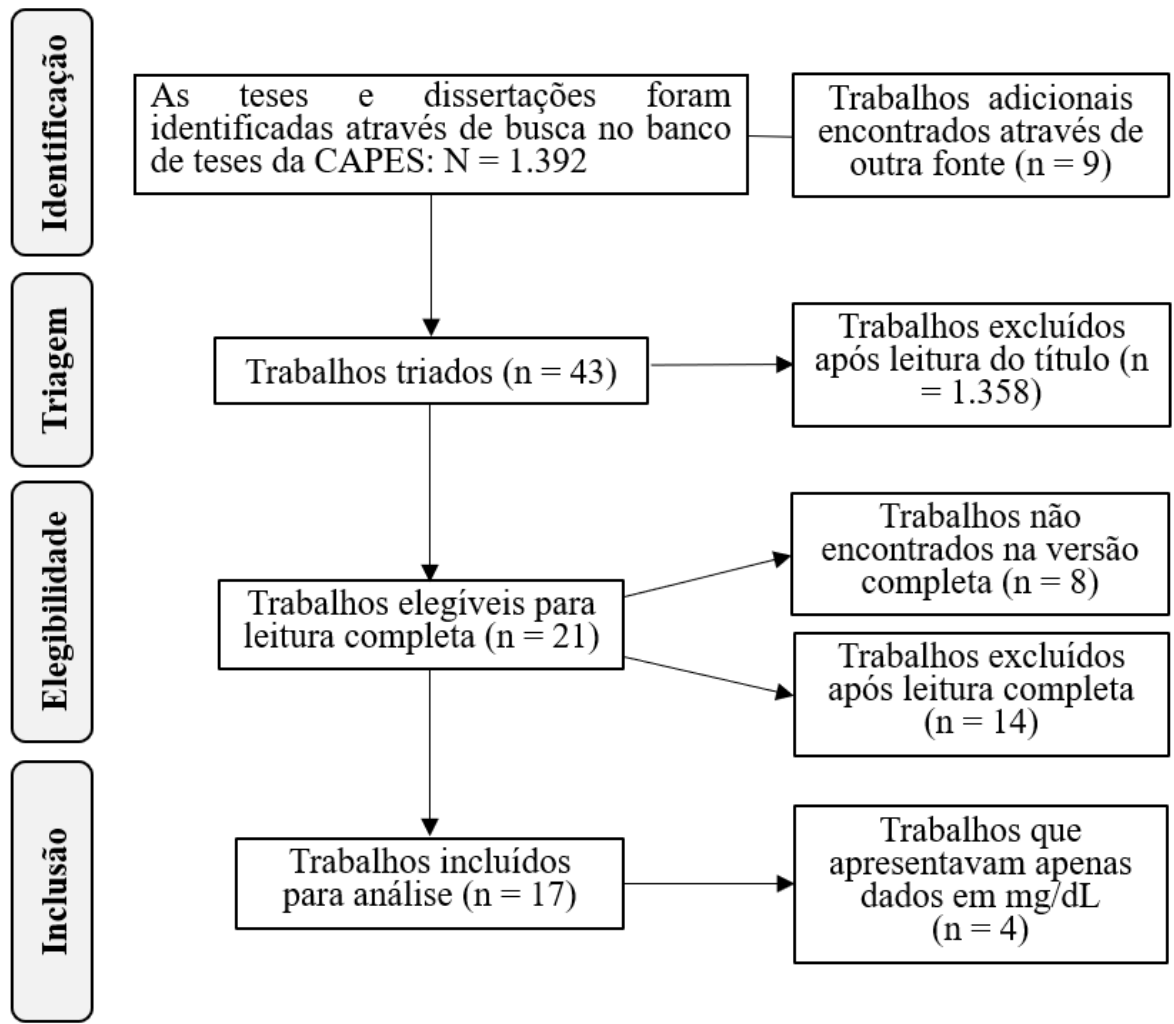

Fonte: Elaborado pelos autores.

A Tabela 1 mostra as características dos estudos incluídos neste trabalho. Foram incluídos 17 trabalhos, com um total de 25.341 participantes, onde pelo menos 11.275 eram 
Research, Society and Development, v. 9, n. 11, e3759119865, 2020

(CC BY 4.0) | ISSN 2525-3409 | DOI: http://dx.doi.org/10.33448/rsd-v9i11.9865

do sexo masculino e 12.658 do sexo feminino. As idades dos participantes variaram entre 0 e 19 anos. Os estudos foram realizados no período entre 2010 a 2020 no Brasil. Sete trabalhos foram realizados na região Sudeste, seis na região Nordeste, dois na região Sul, um na região Centro-Oeste, e um na região Norte.

Tabela 1 - Características dos estudos incluídos no estudo.

\begin{tabular}{|c|c|c|c|c|c|}
\hline \multirow[t]{2}{*}{ Estudo } & \multirow{2}{*}{$\begin{array}{c}\text { Estado } \\
\text { brasileiro }\end{array}$} & \multirow[t]{2}{*}{$\mathbf{N}$} & \multicolumn{2}{|c|}{ Sexo } & \multirow{2}{*}{$\begin{array}{c}\text { Faixa etária } \\
\text { (anos) }\end{array}$} \\
\hline & & & $\begin{array}{l}\text { Masculino } \\
\text { (n) }\end{array}$ & $\begin{array}{c}\text { Feminino } \\
\text { (n) }\end{array}$ & \\
\hline Neto, 2010 & BA & 937 & 469 & 468 & $7-14$ \\
\hline Almeida, 2011 & ES & 511 & 239 & 272 & $6-9$ \\
\hline Lima, 2011 & $\mathrm{RN}$ & 432 & 223 & 209 & $10-19$ \\
\hline Silva, 2013 & MG & 348 & 145 & 203 & $8-9$ \\
\hline Oliveira, 2014 & PR & 53 & - & 53 & $13-17$ \\
\hline \multirow[t]{2}{*}{ Souza, 2014} & RJ & 1.110 & - & - & $2-9(n=607)$ \\
\hline & & & & & $10-19(n=503)$ \\
\hline Saldanha, 2015 & $\mathrm{CE}$ & 232 & 123 & 108 & $6-10$ \\
\hline Maia, 2016 & $\mathrm{CE}$ & 700 & 340 & 360 & $2-9$ \\
\hline Neta, 2019 & PB & 774 & 351 & 423 & $10-14$ \\
\hline Sponchiado, 2017 & $\mathrm{RS}$ & 297 & - & - & $1-6$ \\
\hline Marcelino, 2017 & SP & 10.597 & 5.113 & 5.484 & $0-10$ \\
\hline Oliosa, 2017 & ES & 854 & 478 & 376 & $6-19$ \\
\hline Pires, 2017 & MA & 363 & 202 & 161 & $17-18$ \\
\hline Bauman, 2018 & MG & 635 & 253 & 382 & $10-16$ \\
\hline Polli, 2018 & SP & 299 & 139 & 160 & $5-14$ \\
\hline Silva, 2018 & GO & 158 & 80 & 78 & $4-11$ \\
\hline Costa, 2019 & $\mathrm{AM}$ & 7.041 & 3.120 & 3.921 & $12-17$ \\
\hline TOTAL & & 25.341 & 11.275 & 12.658 & 0 - 19 \\
\hline
\end{tabular}

Fonte: CAPES. 


\subsubsection{Análise do perfil lipídico}

A análise do perfil lipídico dos trabalhos incluídos neste estudo foi realizada de acordo com a V Diretriz Brasileira de Dislipidemias e Prevenção da Aterosclerose vigente no momento dos estudos. Os valores referenciais das variáveis lipídicas considerados como elevados foram: $\mathrm{CT}>170 \mathrm{mg} / \mathrm{dL}$; LDL-c $\geq 130 \mathrm{mg} / \mathrm{dL}$; TG $>130 \mathrm{mg} / \mathrm{dL}$; e considerado abaixo do valor desejável para HDL-c $\leq 45 \mathrm{mg} / \mathrm{dL}$.

A Figura 2A apresenta a frequência de dislipidemia nas amostras da população brasileira. Observa-se que, aproximadamente, 35\% das crianças e adolescentes brasileiros apresentam níveis de CT e HDL-c alterados. Quando esses parâmetros são avaliados por região brasileira (Figura 2B), as amostras da região Norte, Nordeste e Sul apresentam alta taxa (mais que 50\%) de níveis de HDL-c abaixo dos valores considerados desejáveis. Na região Sudeste $47 \%$ e $34 \%$ das crianças e adolescentes estão com níveis de CT e LDL-c, respectivamente, acima dos valores normais. E, na região Centro-Oeste os níveis de TG e CT das amostras estavam 54\% e 35\% mais elevados, respectivamente. Durante a análise do perfil lipídico nas diferentes regiões do Brasil, pode-se observar que o CT e o LDL-c elevados prevalecem na região Sudeste, o TG elevado com maior frequência na região Centro-Oeste e o HDL-c alterado, prevalece nas regiões Norte, Nordeste e Sul.

Através da análise dos dados apresentados nas teses e dissertações incluídas neste trabalho, a hiperlipidemia prevalece na primeira faixa etária (2 a 10 anos), dados demonstrados na Figura 3. As crianças de 2 a 10 anos apresentam maior incidência de CT, TG e LDL-c com valores acima do desejável, quando comparado aos adolescentes. No entanto, $48 \%$ da amostra na faixa etária de 11 a 19 anos apresentavam HDL-c abaixo dos valores normais. 
Figura 2 - Prevalência de dislipidemia nas crianças e adolescentes do Brasil e regiões. A dislipidemia no Brasil; B - distribuição da dislipidemia nas regiões brasileiras. CT: colesterol total (> 170mg/dL); LDL-c: colesterol da lipoproteína de baixa densidade ( $\geq 130 \mathrm{mg} / \mathrm{dL})$; TG: triglicérides (> 130mg/dL); HDL-c: colesterol da lipoproteína de alta densidade $(\leq 45 \mathrm{mg} / \mathrm{dL})$.

A

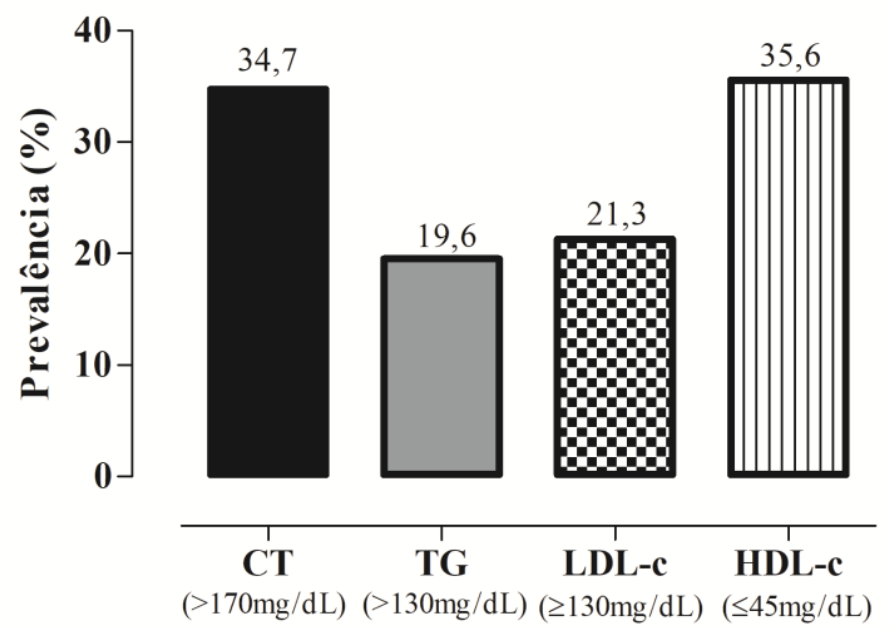

B

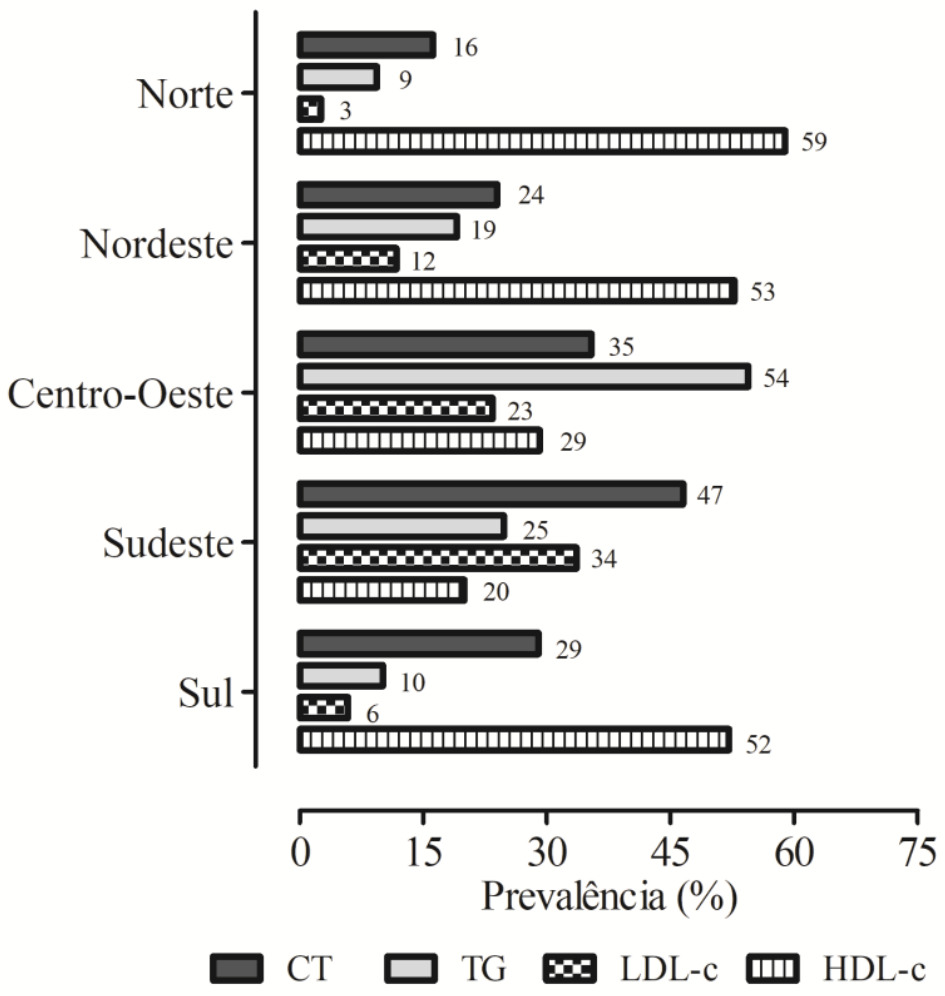

Fonte: Elaborado pelos autores. 
Figura 3 - Prevalência de dislipidemia de acordo com a faixa etária. CT: colesterol total (> 170mg/dL); LDL-c: colesterol da lipoproteína de baixa densidade ( $\geq 130 \mathrm{mg} / \mathrm{dL})$; TG: triglicérides (> 130mg/dL); HDL-c: colesterol da lipoproteína de alta densidade $(\leq 45 \mathrm{mg} / \mathrm{dL})$.

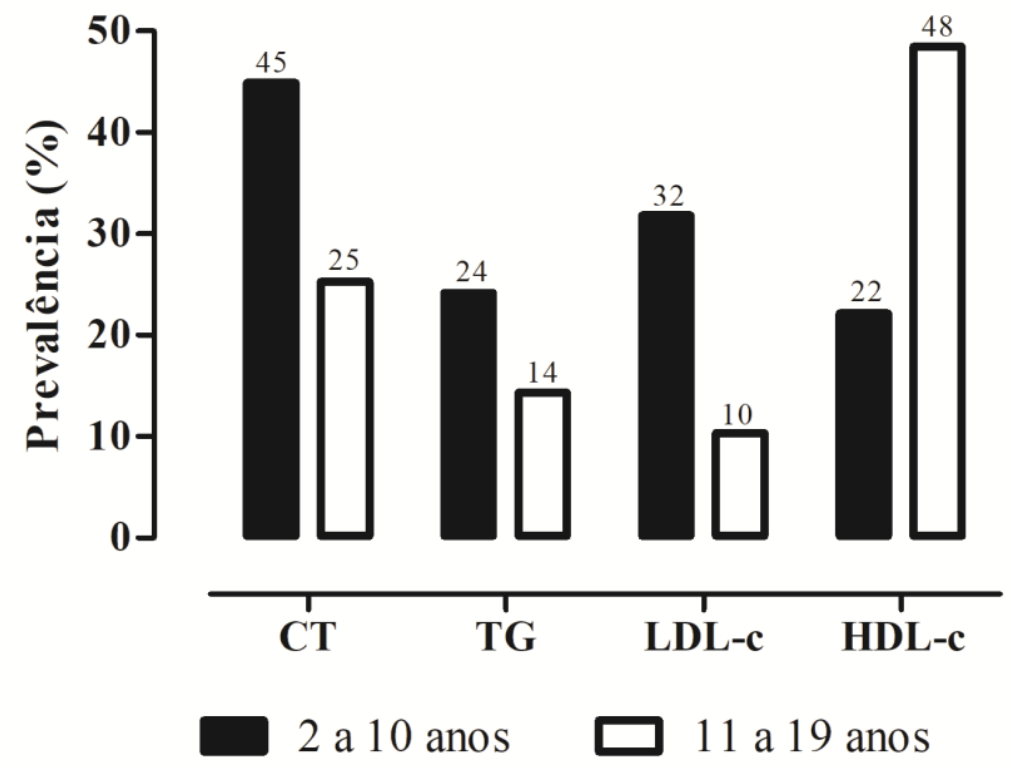

Fonte: Elaborado pelos autores.

\subsection{Análise documental interpretativa da BNCC}

A análise quantitativa realizada neste estudo demonstrou a prevalência das dislipidemias na primeira faixa etária ( 2 a 10 anos), o que parece indicar a necessidade da conscientização de crianças sobre os riscos dos distúrbios lipídicos para a saúde e a importância de mudanças nos hábitos de vida para a redução da prevalência de dislipidemias. Neste caso, a escola pode ter um importante papel na educação dessas crianças através dos componentes curricular de suas disciplinas, em especial as disciplinas de Educação Física, Ciências e Biologia.

Vale enfatizar que a educação em saúde inclui a conscientização dos indivíduos sobre seus direitos e deveres relativos à manutenção da própria saúde e da comunidade na qual se insere A saúde como objeto de formação é um direito público, ligado à qualidade de vida constituindo um dever social (Brasil, 1988). Essa conciliação entre direito público e dever social é fundamental para a educação em saúde. É preciso pensar na escola como um todo inserido na sociedade, pensar na democratização do sucesso (dela e de todos os sujeitos) que passa pela participação de todos os agentes sociais, dentre eles os parceiros, pois "O 
envolvimento dos pais na educação das crianças tem uma justificativa pedagógica e moral, bem como legal [...] Quando os pais iniciam uma parceria com a escola, o trabalho com as crianças pode ir além da sala de aula, e as aprendizagens na escola e em casa possam se complementares mutuamente" (Spodek \& Saracho, 1998, p. 167). Contudo, a educação em saúde deve levar em consideração as reais necessidades, interesses e objetivos de todos que compõem a comunidade escolar ali presente, destacando a importância da participação da família na escola para que a relação entre a comunidade e a escola fique mais estreita, para que haja uma confiança mútua entre as duas instituições e para que o aluno possa se interessar mais pela escola e ter um melhor rendimento.

Pimentel (2001) afirma que pesquisas de natureza de revisão bibliográfica são desenvolvidas por meio de documentos, contemporâneos ou retrospectivos, e têm sido amplamente utilizadas nas Ciências Sociais com a finalidade de descrever e/ou comparar fatos sociais, estabelecendo suas características e tendências.

Outro fator importante é entender que cada criança e adolescente trará conhecimentos prévios independente da disciplina sobre cada assunto que for abordado, mostrando assim a importância de se ouvir os alunos para que o conhecimento aconteça de forma mais natural. De acordo com Young (2007), as disciplinas escolares precisam evidenciar suas fronteiras e delimitá-las também na relação com conhecimentos trazidos pelos estudantes e oriundos da experiência cotidiana; sendo papel da escola o desenvolvimento intelectual dos estudantes por meio das suas disciplinas.

Com isso, surgiu o interesse em analisar o novo documento de orientação para a organização do ensino desde a educação infantil até o ensino médio, para identificar se existem indicações explicitas ou implícitas sobre a abordagem dessa temática e em que etapa da escolaridade ela ocorre, podendo fornecer subsídios para abordagem dessa temática pelos professores.

Neste estudo o documento institucional analisado foi a BNCC, na qual a justificativa se deu pela sua própria definição (Brasil, 2017, p.7):

A Base Nacional Comum Curricular é um documento de caráter normativo que define o conjunto orgânico e progressivo de aprendizagens essenciais que todos os alunos devem desenvolver ao longo das etapas e modalidades da Educação Básica, de modo a que tenham assegurados seus direitos de aprendizagem e desenvolvimento em conformidade com o que preceitua o Plano Nacional de Educação (PNE). 
Durante análise documental da BNCC, homologada em 2017, visando verificar como está sendo abordado e discutido os tópicos sobre risco para saúde e hábitos de vida saudável (alimentação e atividade física) em crianças e adolescentes, foi realizada a leitura completa do documento. A avaliação buscou três fontes de informações, a disciplina de Educação Física, a de Ciências e a de Biologia, uma vez que são as disciplinas onde está prevista a discussão do tema. As competências e habilidades destas disciplinas foram analisadas desde a Educação Infantil até o ensino médio, e são descritas abaixo:

\subsubsection{Educação Infantil}

Segundo a BNCC não foram encontradas habilidades referentes ao tema nas disciplinas de Educação Física e Ciências (Quadro 1).

\subsubsection{Ensino fundamental I e II}

Segundo a BNCC a disciplina Educação Física enfatiza a prática corporal inserida em um contexto social e cultural. Entre os componentes curricular de Educação Física foi analisado as competências específicas que devem ser abordados com os alunos. Entre estas competências específicas relacionadas com o principal tema do presente estudo pode-se destacar (Brasil, 2017, p.223):

Refletir, criticamente, sobre as relações entre a realização das práticas corporais e os processos de saúde/doença, inclusive no contexto das atividades laborais.

Identificar a multiplicidade de padrões de desempenho, saúde, beleza e estética corporal, analisando, criticamente, os modelos disseminados na mídia e discutir posturas consumistas e preconceituosas.

Usufruir das práticas corporais de forma autônoma para potencializar o envolvimento em contextos de lazer, ampliar as redes de sociabilidade e a promoção da saúde.

Embora as competências específicas de Educação Física para o ensino fundamental citem sobre a importância de se usufruir das práticas corporais como forma de promoção da saúde, não é previsto durante todo o ensino fundamental I um tópico que aborde esse tema. Somente no ensino fundamental II, após o $6^{\circ}$ ano que se tem a presença de tópicos curriculares que associam a prática de exercícios físicos com melhorias da saúde (Quadro 1).

Além disso, também foi avaliado na BNCC, através da análise na área de Ciências da Natureza, dos componentes curriculares de Ciências a existência de pontos relacionados a 
promoção da saúde. Entre as competências específicas relacionadas com o principal tema do presente estudo pode-se destacar (Brasil, 2017, p.324): “Conhecer, apreciar e cuidar de si, do seu corpo e bem-estar, compreendendo-se na diversidade humana, fazendo-se respeitar e respeitando o outro, recorrendo aos conhecimentos das Ciências da Natureza e às suas tecnologias."

Os resultados mostram que durante o ensino fundamental $\mathrm{I}$, somente no $5^{\circ}$ ano as crianças aprendem sobre os distúrbios nutricionais causados pelos hábitos de vida (Quadro 1). Ou seja, somente quando a criança está com 10 a 11 anos de idade esse tema é abordado. E, no ensino fundamental II, há somente um tópico no $7^{\circ}$ ano que cita a necessidade de se interpretar as condições de saúde da comunidade e dos resultados de políticas públicas destinadas à saúde (Quadro 1).

Quadro 1 - Avaliação dos tópicos das disciplinas de Educação Física e Ciências extraídas da BNCC (Brasil, 2017), segundo a presença de componentes que realizem promoção de saúde no ensino Fundamental e Médio.

\begin{tabular}{|c|c|c|}
\hline $\begin{array}{l}\text { ENSINO } \\
\text { BÁSICO }\end{array}$ & DISCIPLINA/ÁREA & HABILIDADES \\
\hline \multirow[t]{2}{*}{$1^{\circ}-4^{\circ}$ ano } & Educação Física & Nada consta. \\
\hline & Ciências & Nada consta. \\
\hline \multirow[t]{2}{*}{$5^{\circ}$ ano } & Educação Física & Nada consta. \\
\hline & Ciências & $\begin{array}{l}\text { (EF05CI08) Organizar um cardápio equilibrado com base } \\
\text { nas características dos grupos alimentares (nutrientes e } \\
\text { calorias) nas necessidades individuais (atividades } \\
\text { realizadas, idade, sexo etc.) para a manutenção da saúde do } \\
\text { organismo (p.341). } \\
\text { (EF05CI09) Discutir a ocorrência de distúrbios } \\
\text { nutricionais (como obesidade, subnutrição etc.) entre } \\
\text { crianças e jovens a partir da análise de seus hábitos (tipos e } \\
\text { quantidade de alimento ingerido, prática de atividade física } \\
\text { etc.) (p.341). }\end{array}$ \\
\hline \multirow[t]{2}{*}{$6^{\circ}$ ano } & Educação Física & $\begin{array}{l}\text { (EF67EF09) Construir, coletivamente, procedimentos e } \\
\text { normas de convívio que viabilizem a participação de todos } \\
\text { na prática de exercícios físicos, com o objetivo de } \\
\text { promover a saúde (p.233). }\end{array}$ \\
\hline & Ciências & Nada consta. \\
\hline $7^{\circ}$ ano & Educação Física & $\begin{array}{l}\text { (EF67EF10) Diferenciar exercício físico de atividade física } \\
\text { e propor alternativas para a prática de exercícios físicos }\end{array}$ \\
\hline
\end{tabular}


Research, Society and Development, v. 9, n. 11, e3759119865, 2020

(CC BY 4.0) | ISSN 2525-3409 | DOI: http://dx.doi.org/10.33448/rsd-v9i11.9865

\begin{tabular}{|c|c|c|}
\hline & & dentro e fora do ambiente escolar. (p.233). \\
\hline & Ciências & $\begin{array}{l}\text { (EF07CI09) Interpretar as condições de saúde da } \\
\text { comunidade, cidade ou estado, com base na análise e } \\
\text { comparação de indicadores de saúde (como taxa de } \\
\text { mortalidade infantil, cobertura de saneamento básico e } \\
\text { incidência de doenças de veiculação hídrica, atmosférica } \\
\text { entre outras) e dos resultados de políticas públicas } \\
\text { destinadas à saúde (p.347). }\end{array}$ \\
\hline $8^{\circ}$ ano & Educação Física & $\begin{array}{l}\text { (EF89EF07) Experimentar e fruir um ou mais programas } \\
\text { de exercícios físicos, identificando as exigências corporais } \\
\text { desses diferentes programas e reconhecendo a importância } \\
\text { de uma prática individualizada, adequada às características } \\
\text { e necessidades de cada sujeito (p.237). }\end{array}$ \\
\hline & Ciências & Nada consta. \\
\hline $9^{\circ}$ ano & Educação Física & $\begin{array}{l}\text { (EF89EF11) Identificar as diferenças e semelhanças entre } \\
\text { a ginástica de conscientização corporal e as de } \\
\text { condicionamento físico e discutir como a prática de cada } \\
\text { uma dessas manifestações pode contribuir para a melhoria } \\
\text { das condições de vida, saúde, bem-estar e cuidado consigo } \\
\text { mesmo (p.237). }\end{array}$ \\
\hline & Ciências & Nada consta. \\
\hline Médio & Educação Física & $\begin{array}{l}\text { (EM13LGG503) Vivenciar práticas corporais e significá- } \\
\text { las em seu projeto de vida, como forma de } \\
\text { autoconhecimento, autocuidado com o corpo e com a } \\
\text { saúde, socialização e entretenimento (p.495). }\end{array}$ \\
\hline & Ciências da Natureza & $\begin{array}{l}\text { (EM13CNT310) Investigar e analisar os efeitos de } \\
\text { programas de infraestrutura e demais serviços básicos } \\
\text { (saneamento, energia elétrica, transporte, } \\
\text { telecomunicações, cobertura vacinal, atendimento primário } \\
\text { à saúde e produção de alimentos, entre outros) e identificar } \\
\text { necessidades locais e/ou regionais em relação a esses } \\
\text { serviços, a fim de avaliar e/ou promover ações que } \\
\text { contribuam para a melhoria na qualidade de vida e nas } \\
\text { condições de saúde da população (p.560). }\end{array}$ \\
\hline
\end{tabular}

Fonte: Elaborado pelos autores. Dados extraídos da BNCC.

\subsubsection{Ensino Médio}

Na BNCC a Educação Física no Ensino Médio está inserida dentro da área de Linguagens e suas Tecnologias. No Ensino Médio são aprofundados os conhecimentos sobre 
os limites do corpo, a importância de se ter um estilo de vida ativo e à manutenção da saúde. Durante o Ensino Médio, dentro da Área de Linguagens, existe apenas um único tópico que descreve sobre as práticas corporais em prol da saúde (Quadro 1).

Já na Área de Ciências da Natureza a abordagem sobre melhoria na qualidade de vida e promoção da saúde foi citada também em apenas um tópico (Quadro 1).

\section{Discussão}

Nos últimos anos têm ocorrido cada vez mais discussões sobre aumento nos níveis séricos de lipídeos, a obesidade, e as doenças cardiovasculares. Estes assuntos trazem à tona um importante problema de saúde pública, as dislipidemias. Esse problema vêm atingindo um número cada vez maior da população nacional e internacional, apresentando uma prevalência que varia entre $2,8 \%$ a 46,8\%, com aumento proporcional à idade entre adolescentes (FariaNeto et al., 2016; Kit et al., 2015). A dislipidemia na infância tem apontado diversos fatores de riscos, principalmente nas doenças cardiovasculares, como o acidente vascular cerebral e o infarto agudo do miocárdio (Pereira et al., 2013). São classificadas como doenças crônicas não transmissíveis e constituem o problema de saúde de maior magnitude, correspondendo a cerca de $70 \%$ das causas de mortes, atingindo fortemente camadas pobres da população e grupos mais vulneráveis, como a população de baixa escolaridade e renda (Malta et al., 2020).

No presente estudo o diagnóstico situacional da dislipidemia demonstrou que crianças e adolescentes (2-19 anos) apresentaram alterações com relação ao perfil lipídico que propiciam um potencial risco para o desenvolvimento de doença cardiovascular na vida adulta, e que as diretrizes para abordagem sobre o assunto nos âmbitos escolares ocorrem de forma discreta e somente após a idade na qual os hábitos alimentares já estão estabelecidos.

No estudo quantitativo através de revisão integrativa foi mostrada a prevalência de $35 \%$ dos níveis elevados de CT, 20\% de TG e 21\% de LDL-c, além dos níveis baixos de HDL-c em $36 \%$ da população estudada. Segundo Cook \& Rae (2011) o padrão de dislipidemia associado à obesidade pediátrica consiste em uma combinação de TG elevados, HDL-c reduzido e LDL-c superior ao normal ou levemente elevado. Estes dados indicam que a dislipidemia está presente em pelo menos 35\% da população dos brasileiros de 2 a 19 anos, ou seja, 1/3 da população jovem brasileira está com seu perfil lipídico alterado. Resultados semelhantes também foram observados por Gomes et al (2020), que avaliaram na região Sudeste indivíduos entre 2 e 19 anos $(n=61.870)$, e perceberam que destes, $33 \%$ de CT, $40 \%$ 
de TG, $29 \%$ de LDL-c estavam com os níveis séricos elevados e 39\% de HDL-c abaixo do desejado.

Ao analisar a dislipidemia nas diferentes regiões do Brasil foi observado que mais de $50 \%$ da população estudada nas regiões Norte, Nordeste e Sul apresentavam níveis de HDL-c abaixo dos valores indicados. Faria-Neto et al (2016) também observaram resultados semelhantes quanto à prevalência da hipolipidemia nas regiões Norte $(58,7 \%)$ e Nordeste (51,6\%). Enquanto Cunha et al (2018) observou valores similares na região Sul (52\%). Ambos os trabalhos foram realizados com população jovem entre 10 e 19 anos. Já nas regiões Sudeste e Centro-Oeste a hiperlipidemia prevaleceu. Na região Sudeste, mais de $34 \%$ dos jovens apresentavam níveis de CT e LDL-c elevados. No estudo realizado por Gomes et al (2020) semelhantes resultados foram encontrados com crianças e adolescentes na região sudeste, CT (33\%) e LDL-c (29\%). Na região Centro-Oeste os dados apontam que mais de $50 \%$ dos indivíduos estavam com níveis de TG acima do normal. Nesta região, apenas 1 trabalho (Silva, 2018) se enquadrou nos critérios de inclusão, então os dados podem não ser significativos devido à pequena amostra $(n=158)$. No entanto, os resultados são importantes para que novas investigações sejam realizadas nessa população visto que níveis elevados de TG podem levar ao desenvolvimento de doenças cardíacas (Cook \& Rae, 2011). Assim, o monitoramento do perfil lipídico é necessário e auxilia na elaboração de estratégias de controle para evitar que doenças cardiovasculares se estabeleçam na idade adulta. A divergência dos resultados de acordo com cada região do Brasil pode estar relacionada aos diferentes hábitos alimentares, genética, idade, estilo de vida, entre outros. Segundo Alcântara Neto et al (2012), em estudo realizado com indivíduos entre 7 e 14 anos na região Nordeste, o excesso de peso, consumo alimentar inadequado e baixa escolaridade materna podem ser os fatores associados a dislipidemia, principalmente nesta região.

Outro ponto importante foi a avaliação nas diferentes faixas etárias (2 a 10 anos e 11 a 19 anos). No presente trabalho, os resultados mostraram a presença da hiperlipidemia nas crianças de 2 a 10 anos (Figura 3). Já nos adolescentes de 11 a 19 anos a hipolipidemia (HDLc baixo) se destaca. Resultados semelhantes foram encontrados em estudo realizado na região Sudeste onde os níveis elevados de CT (40\%), LDL-c (35\%) e TG (45\%) prevaleceram em crianças de 2 a 11 anos, e baixos níveis de HDL-c prevaleceram em $41 \%$ dos adolescentes de 12 a 19 anos (Gomes et al., 2020). Outro estudo realizado com adolescentes entre 10 e 19 anos na região Sul também mostrou que a hipolipidemia estava presente em $52 \%$ dos indivíduos (Cunha et al., 2018). Estes fatos corroboram os relatos presentes na I Diretriz de Prevenção da Aterosclerose na Infância e na Adolescência (Giuliano et al., 2005), na qual é 
citado que os níveis séricos de lipídeos e lipoproteínas podem sofrer interferências de acordo com a idade e o sexo durante o crescimento e desenvolvimento das crianças e adolescentes. Além disso, os níveis de lipídeos e lipoproteínas se encontram mais elevados nas crianças. Outro ponto importante que poderia influenciar essas mudanças no perfil lipídico é a fase do pico de crescimento. No entanto, alguns estudos mostram que a presença do hormônio de crescimento promove melhora no perfil lipídico (Chen et al., 2018; Kubo et al., 2018). Assim, como em nosso estudo ainda observamos a prevalência da dislipidemia em jovens acima de 10 anos, quando o quadro dislipidêmico seria bem pior se não houvesse os picos de GH normais para a idade.

Atualmente no Brasil se observa uma diminuição na desnutrição infantil, no entanto, simultaneamente o país vem aumentando de forma expressiva o sobrepeso e a obesidade em todas as faixas etárias. Diante desse cenário, que impactou nas condições de saúde e nutrição da sociedade brasileira, o Ministério da Saúde apresentou em 2014 novas recomendações sobre alimentação saudável através do Guia Alimentar para a População Brasileira (Brasil, 2014). Essa demonstração da relação da alimentação equilibrada e seus benefícios à saúde também são discutidos em diversos estudos, como o de Saeedi et al (2019) que mostram a importância de hábitos de alimentação equilibrada na infância e a presença de marcadores de saúde cardiovascular como benefício na redução de risco de doença cardiovascular tardia.

Diante dessa problemática, intervenções profiláticas através da adoção de medidas preventivas das doenças cardiovasculares, visto que a formação dos hábitos individuais ocorre deste a infância é de suma importância. Estudos longitudinais, como o de Juonala et al (2011), demonstram que são mais efetivas as prevenções das doenças cardiovasculares em adultos, quando se tem alguma intervenção enquanto criança. Segundo a I Diretriz de Prevenção da Aterosclerose na Infância e Adolescência as modificações de hábitos e preferências alimentares introduzidas na infância podem se tornar permanentes (Giuliano et al., 2005). Uma ótima forma de disseminar tal informação para crianças e adolescentes é através do ensino escolar. Dessa forma, no presente trabalho buscou-se analisar as diretrizes preconizadas na BNCC sobre esse tema (Brasil, 2017).

Durante a análise da BNCC, não foram encontradas habilidades referentes ao tema na Educação Infantil e no Ensino Fundamental I, nas principais disciplinas relacionadas às práticas para a promoção da saúde, Educação Física e Ciências.

A disciplina de Educação Física na Educação Infantil é inserida na categoria de Corpo/Movimento e Jogo/Brincadeira pois são as categorias que estabelecem maior interface com os objetivos de estudo da Educação Física junto ao documento norteador que é o 
Referencial Curricular Nacional da Educação Infantil (RCNEI). O RCNEI afirma que o trabalho pedagógico na Educação Infantil deve contemplar “[...] a multiplicidade de funções e manifestações do ato motor, propiciando um amplo desenvolvimento de aspectos específicos da motricidade das crianças, [...] bem como atividades voltadas para a ampliação da cultura corporal” (Brasil, 1998, p.15). O documento ainda apresenta um caráter instrumental do corpo/movimento, cuja função primordial da ação motora na Educação Infantil é oferecer suporte para futuras aprendizagens.

$\mathrm{Na} \mathrm{BNCC}$, os processos pedagógicos na Educação Infantil partem da concepção de que a construção de conhecimento pelas crianças se efetiva por meio da participação direta delas nas diferentes práticas cotidianas.

De acordo com Sayão (1999, p.224), ao discutir a presença da Educação Física na Educação Infantil, destaca a necessidade de se compreender as especificidades dos indivíduos criança e focaliza o "[...] movimento corporal como instrumento de apropriação das diferentes linguagens produzidas pela cultura que é reinventada pelas crianças”.

$\mathrm{Na}$ disciplina de Ciências na Educação Infantil o corpo humano é trabalhado nas escolas de forma fragmentada seguindo esquemas, sistemas, compartimentos, onde cada órgão é classificado segundo sua função. Segundo Rabello (1994) a organização pedagógica não interligada dificulta o entendimento da criança a respeito do seu corpo e a compreensão de que todos os seus órgãos estão integrados formando um sistema único. Além disso, o estudo do corpo humano é um incentivo as crianças. Leva elas a questionar e voltar seu olhar para seu próprio corpo que está constantemente sofrendo mudanças. Com isso, compete ao professor usufruir destes momentos para perceber o grau de conhecimento do corpo humano seus alunos têm e, a partir de então, conduzir seu ensino com diretrizes que melhor esclarecem as dúvidas de seus alunos.

Somente após o $6^{\circ}$ ano (ensino fundamental II) que se tem a presença de tópicos curriculares que associam a prática de exercícios físicos com melhoria da saúde. Já na disciplina de Ciências, os resultados mostram que somente a partir do $5^{\circ}$ ano as crianças aprendem sobre os distúrbios nutricionais causados pelos hábitos de vida. Os dados quantitativos de alterações no perfil lipídico de crianças e adolescentes apresentados neste trabalho só reforçam a necessidade da mudança de hábitos de vida deste a infância, como já citado também em outros estudos (Bays et al., 2016; Brown, 2018). Isso significa que, seria de grande importância melhorar a base curricular do $1^{\circ}$ ao $4^{\circ}$ ano do ensino fundamental, inserindo a abordagem de hábitos saudáveis para crianças a partir dos 5 anos. Além disso, não há a preocupação em demonstrar que existe uma relação entre o se movimentar e a promoção 
da saúde nessa faixa etária, ficando a carácter do professor mostrar tais problemas, em que poderiam contribuir para a conscientização e prevenção de doenças na fase adulta. Burchard et al (2020) evidenciaram uma fragilidade do trabalho interdisciplinar e contextualizado da prática pedagógica na temática saúde através da análise da BNCC, sendo que este tema ainda está confinado aos componentes curriculares de Ciências e Educação Física, sendo rara a utilização do termo ou do conceito em outros componentes curriculares.

A ausência de conhecimentos científicos bem estruturados nas disciplinas como de Educação Física, Ciências e Biologia torna o ato de ensinar um desafio. São com estes conhecimentos científicos que constantemente buscamos esclarecer e verificar os fatos existentes. Diante desta inquietude é que o educador pode abordar com clareza a importância desse conhecimento aos seus alunos. Despertando assim, uma curiosidade do público-alvo e tornando suas aulas mais interessantes (Viecheneski \& Carletto, 2012). Contudo, podemos dizer que o conhecimento de educação em saúde, articulado ao Ensino de Ciências, através da orientação sobre hábitos de vida saudáveis, poderá contribuir para fortalecer ações que promovam a saúde das crianças e adolescentes, levando a formação de cidadãos mais conscientes.

\section{Considerações Finais}

Neste estudo constatou-se que é alta a prevalência da dislipidemia em crianças e adolescentes de todas as regiões brasileiras, propiciando um potencial risco para o desenvolvimento de doença cardiovascular na vida adulta. Além disso, as diretrizes de orientação do tema nos âmbitos escolares ocorrem de forma discreta e somente após a idade na qual os hábitos individuais já estão estabelecidos, e deve-se levar em consideração as reais necessidades, interesses e objetivos de todos que compõem a comunidade escolar ali presente, destacando a importância da participação da família para que a relação entre a comunidade e a escola fique mais estreita, permitindo uma maior conscientização dos indivíduos sobre seus direitos e deveres relativos à manutenção da própria saúde e da comunidade na qual se insere. Sugere-se que o conhecimento científico articulado ao Ensino de Ciências, deve estimular o processo de percepção dos educandos sobre a importância de uma rotina de vida fisicamente ativa e a implantação de hábitos alimentares saudáveis, para a promoção da saúde.

\section{Referências}

Alcântara Neto, O. D. de, Silva, R. de C. R., Assis, A. M. O., \& Pinto, E. D. J. (2012). Fatores 
Research, Society and Development, v. 9, n. 11, e3759119865, 2020

(CC BY 4.0) | ISSN 2525-3409 | DOI: http://dx.doi.org/10.33448/rsd-v9i11.9865

associados à dislipidemia em crianças e adolescentes de escolas públicas de Salvador, Bahia Factors associated with dyslipidemia in children and adolescents enrolled in public schools of Salvador, Bahia. Rev Bras Epidemiol, 15(2), 335-345. https://doi.org/10.1590/S0102311X2009000400019

Almeida, D. F., Júnior, W. A. (2016). Base Nacional de Educação Física: Faz sentindo a ideia de um currículo nacional? VI Seminário de Metodologia de Ensino de Educação Física.

Almeida, P. C. D. de. (2011). Alterações antropométricas e metabólicas em escolares de seis a nove anos do município de Vitória - ES. Universidade Federal de Minas Gerais. 63p.

Bauman, C. D. (2018). Prevalência de dislipidemia em adolescentes da rede pública de ensino estadual da cidade de Montes Claros - MG. Universidade Estadual de Montes Claros. $135 \mathrm{p}$.

Bays, H. E., Jones, P. H., Orringer, C. E., Brown, W. V., \& Jacobson, T. A. (2016). National Lipid Association Annual Summary of Clinical Lipidology 2016. Journal of Clinical Lipidology, 10(1), S1-S43. https://doi.org/10.1016/j.jacl.2015.08.002

Benefield, L. E. (2003). Implementing Evidence-Based Practice in Home Care. Home Healthc Nurse, 21(12), 804-811. https://pubmed.ncbi.nlm.nih.gov/14665967/

Beyea, Suzanne C; Nicoll, L. H. (1998). Writing an integrative review. AORN Journal, 67(4), 877-880. https://www.sciencedirect.com/science/article/pii/S0001209206626537

Brasil. (1988). Constituição da República Federativa do Brasil. Brasília: Centro Gráfico. 413p.

Brasil. (1998). Conhecimento De Mundo. Referencial curricular nacional para a educação infantil - Conhecimento de Mundo, 3, 117-157.

Brasil. (2014). Guia alimentar para a população brasileira (2 ed). Brasília: Ministério da Saúde. 158p. 
Research, Society and Development, v. 9, n. 11, e3759119865, 2020

(CC BY 4.0) | ISSN 2525-3409 | DOI: http://dx.doi.org/10.33448/rsd-v9i11.9865

Brasil. (2017). Base Nacional Curricular Comum - BNCC. Mec, 600. https://doi.org/10.1017/CBO9781107415324.004

Brown, W. V. (2018). Clinical Lipidology and the Prevention of Vascular Disease: Time for Personalized Therapy. Clinical Pharmacology and Therapeutics, 104(2), 269-281. https://doi.org/10.1002/cpt.1127

Burchard, CP; Soares, RG; Vargas, VC; Ilha, PV; Ruppenthal, R. (2020). Analysis of the health theme in the base nacional comum curricular. Research, Society and Development, $9(7), 1-14$.

Campos, C. G., Muniz, L. A., Belo, V. S., Romano, M. C. C., \& De Castro Lima, M. (2019). Adolescents' knowledge about the benefits of physical exercises to mental health. Ciencia $e$ Saude Coletiva, 24(8), 2951-2958. https://doi.org/10.1590/1413-81232018248.17982017

Castro Cabezas, M., Burggraaf, B., \& Klop, B. (2018). Dyslipidemias in clinical practice. Clinica Chimica Acta, 487(May), 117-125. https://doi.org/10.1016/j.cca.2018.09.010

Chen, M., Gan, D., Luo, Y., Rampersad, S., Xu, L., Yang, S., Li, N., \& Li, H. (2018). Effect of recombinant human growth hormone therapy on blood lipid and carotid intima-media thickness in children with growth hormone deficiency. Pediatric Research, 83(5), 954-960. https://doi.org/10.1038/pr.2017.271

Cook, S., \& Rae, E. W. K. (2011). Dyslipidemia and pediatric obesity. Pediatric Clinics of North America, 58(6), 1363-1373. https://doi.org/10.1016/j.pcl.2011.09.003

Cooper, H. (1984). The integrative research review: a systematic approach. Sage Pulications.

Costa, M. S. (2019). Consumo alimentar em adolescentes na região norte e fatores associados. Universidade Federal do Amazonas. 141p.

Cunha, E. del B. B., Fagundes, R. P., Scalabrin, E. E., \& Herai, R. H. (2018). Evaluation of Lipid Profile in Adolescents. International Journal of Cardiovascular Sciences, 31(4), 367373. https://doi.org/10.5935/2359-4802.20180034 
Faria-Neto, J. R., Bento, V. F. R., Baena, C. P., Olandoski, M., De Oliveira Gonçalves, L. G., De Azevedo Abreu, G., Kuschnir, M. C. C., \& Bloch, K. V. (2016). ERICA: Prevalence of dyslipidemia in Brazilian adolescents. Revista de Saude Publica, 50(supl 1), 1s-10s. https://doi.org/10.1590/S01518-8787.2016050006723

Ferreira, M. J., Irigoyen, M. C., Consolim-Colombo, F., Saraiva, J. F. K., \& De Angelis, K. (2020). Vida Fisicamente Ativa como Medida de Enfrentamento ao COVID-19. Arquivos Brasileiros de Cardiologia, 601-602. https://doi.org/10.36660/abc.20200235

Ganong, L. H. (1987). Integrative reviews of nursing research. Research in Nursing \& Health, 10(1), 1-11. https://doi.org/10.1002/nur.4770100103

Giuliano, I. de C. B., Caramelli, B., Pellanda, L., Duncan, B., Mattos, S., \& Fonseca, F. H. (2005). I Diretriz de Prevenção da Aterosclerose na Infância e na Adolescência. Arquivos Brasileiros de Cardiologia, 85(Suplemento VI), 1-36.

Gomes, É. I. L., Zago, V. H. de S., \& de Faria, E. C. (2020). Evaluation of lipid profiles of children and youth from basic health units in Campinas, SP, Brazil: A cross-sectional laboratory study. Arquivos Brasileiros de Cardiologia, 114(1), 47-56. https://doi.org/10.5935/abc.20190209

Guedes, D. P., \& Guedes, J. E. R. P. (1995). Atividade física, aptidão física e saúde. Revista Brasileira de Atividade Física e Saúde, 1(1), 18-35.

Juonala, M., Magnussen, C. G., Berenson, G. S., Venn, A., Burns, T. L., Sabin, M. A., Srinivasan, S. R., Daniels, S. R., Davis, P. H., Chen, W., Sun, C., Cheung, M., Viikari, J. S. A., Dwyer, T., \& Raitakari, O. T. (2011). Childhood adiposity, adult adiposity, and cardiovascular risk factors. New England Journal of Medicine, 365(20), 1876-1885. https://doi.org/10.1056/NEJMoa1010112

Kit, B. K., Kuklina, E., Carroll, M. D., Ostchega, Y., Freedman, D. S., \& Ogden, C. L. (2015). Prevalence of and trends in dyslipidemia and blood pressure among us children and adolescents, 1999-2012.

$J A M A$

Pediatrics,

$169(3)$, $272-279$. 
https://doi.org/10.1001/jamapediatrics.2014.3216

Kubo, T., Furujo, M., Takahashi, K., Hyodo, Y., Tsuchiya, H., Hattori, M., Fujinaga, S., \& Urayama, K. (2018). Effects of Growth Hormone Treatment on Lipid Profiles. Indian Journal of Pediatrics, 85(4), 261-265. https://doi.org/10.1007/s12098-017-2509-8

Lima, S. C. V. C. (2011). Estado nutricional antropométrico, perfil lipídico e consumo alimentar como fatores de risco para doença cardiovascular em adolescentes. Universidade Federal do Rio Grande do Norte. 131p.

Maia, J. A. D. F. (2016). Perfil lipídico em crianças de dois a nove anos de idade: construção e validação de manual de cuidados. Universidade Estadual do Ceará. 167p.

Malta, D. C., Silva, A., Cardoso, L. S. de M., Andrade, F. M., Sá, A. C. M. G., Prates, E. J., Alves, F. T., \& Junior, G. F. X. (2020). Doenças Crônicas Não Transmissíveis na revista Ciência \& Saúde Coletiva: Um estudo bibliométrico. Ciência \& Saúde Coletiva. http://www.cienciaesaudecoletiva.com.br/artigos/doencas-cronicas-nao-transmissiveis-narevista-ciencia-saude-coletiva-um-estudo-bibliometrico/17624?id=17624

Marcelino, E. Y. (2017). Dislipidemia na infância: levantamento em crianças da região do Alto Tietê - São Paulo. Universidade de Mogi das Cruzes. 84p.

Martins, I. S., Coelho, L. T., Matos, I. M., Mazzilli, R. N., Trigo, M., \& Wilson, D. (1989). Dislipidemias e alguns fatores de risco associados em uma população periférica da Região Metropolitana de São Paulo, SP-Brasil. Um estudo piloto. Revista de saude publica, 23(3), 236-243. https://doi.org/10.1590/s0034-89101989000300009

Matsudo, S. M., Matsudo, V. H. R., \& Neto, T. L. B. (2012). Efeitos Benéficos Da Atividade Física Na Aptidão Física E Saúde Mental Durante O Processo De Envelhecimento. In Revista Brasileira de Atividade Física e Saúde. 5(2),. 60-76. https://doi.org/10.12820/rbafs.v.5n2p6076

Mendes, K. D. S., Silveira, R. C. de C. P., \& Galvão, C. M. (2008). Revisão integrativa: método de pesquisa para a incorporação de evidências na saúde e na enfermagem. Texto \& 
Neta, A. da C. P. de A. (2019). Padrões alimentares e fatores de risco cardiovasculares em adolescentes. Universidade de São Paulo. 100p.

Neto, O. D. de A. (2010). Fatores associados à dislipidemia em crianças e adolescentes de escolas públicas de Salvador, Bahia, Brasil. Universidade Federal da Bahia. 97p.

Oliosa, P. R. (2017). Associação entre composição corporal, estágio puberal, hábitos de vida e dislipidemia em crianças e adolescentes. Universidade Federal do Espírito Santo. 83p.

Oliveira, P. R. C. (2014). Associação entre adiposidade, parâmetros metabólicos e marcadores inflamatórios em adolescentes do sexo feminino. Universidade Federal do Paraná. 85p.

Pereira, J. A., Rondó, P. H. C., Lemos, J. O., de Oliveira, E., Rocha, C., \& Hipó, T. (2013). Nutritional status and lipid profile of young children in Brazil. Journal of Tropical Pediatrics, 59(1), 54-58. https://doi.org/10.1093/tropej/fms035

Pimentel, A. (2001). O método da análise documental: seu uso numa pesquisa historiográfica. Cadernos de Pesquisa, 114, 179-195. https://doi.org/10.1590/s0100-15742001000300008

Pires, B. R. F. (2017). Padrão alimentar e dislipidemia em adolescentes de escolas públicas de uma capital do nordeste brasileiro. Universidade Federal do Maranhão. 101p.

Polit, Denise F. Cheryl; Beck, T. (2006). The Content Validity Index: Are You Sure You Know What's Being Reported? Critique and Recommendations. Research in Nursing \& Health, 29, 489-497.

Polli, C. A. (2018). Obesidade e sobrepeso: frequência de comorbidades em crianças atendidas nas UBS Central, Guiomar, Monte Serrat, Santa Fé - Itupeva e CE- Jundiaí, SPJundiaí. Faculdade de Medicina de Jundiaí. 79p.

Rabello, S. H. D. S. (1994). A Criança, seu Corpo, suas Idéias. Ensino Em Re-Vista, 3(1), 15- 
29.

Redeker, N. S. (2000). Sleep in acute care settings: An integrative review. Journal of Nursing Scholarship, 32(1), 31-38. https://doi.org/10.1111/j.1547-5069.2000.00031.x

Saeedi, P., Shavandi, A., \& Skidmore, P. M. L. (2019). What do we know about diet and markers of cardiovascular health in children: A review. International Journal of Environmental Research and Public Health, 16(4). https://doi.org/10.3390/ijerph16040548

Saldanha, G. B. (2015). Determinantes de risco associados às dislipidemias em crianças. Universidade Estadual do Ceará. 133p.

Sayão, D. T. (1999). Educacfio Fisica na educacfio infantil: riscos, conflitos e controversias. Motrivivência, 13, 221-238.

Shattat, G. F. (2014). A review article on hyperlipidemia: Types, treatments and new drug targets. Biomedical and Pharmacology Journal, 7(2), 399-409. https://doi.org/10.13005/bpj/504

Silva, A. R. (2013). Componentes da síndrome metabólica e fatoresassociados em crianças de 8 e 9 anos do município de Viçosa- MG. Universidade Federal de Viçosa. 104p.

Silva, D. A. (2018). Prevalência de fatores de risco cardiovascular agrupados em escolares. Universidade Federal de Goiás. 74p.

Silva, R. S., Silva, I., Azevedo, R., Souza, L., \& Tomasi, E. (2010). Atividade física e qualidade de vida Physical activity and quality of life. Ciência \& Saúde Coletiva, 15(1), 115120.

Souza, M. T. de, Silva, M. D. da, \& Carvalho, R. de. (2010). Revisão integrativa: o que é e como fazer. Einsten, 8, 102-106.

Souza, I. dos S. (2014). História familiar para fatores de risco cardiovascular e perfil lipídico e antropométrico em crianças e adolescentes: estudo transversal em unidade ambulatorial do 
Rio de Janeiro. Escola Nacional de Saúde Pública Sérgio Arouca. 88p.

Spodek, B.; Saracho, O. N. (1998). Ensinando crianças de 3 a 8 anos. ArtMed.

Sponchiado, F. C. (2017). Quais fatores estão associados ao excesso de peso e à dislipidemia em crianças menores de 7 anos num pequeno município do interior caracteristicamente rural? Universidade Federal do Rio Grande do Sul. 58p.

Stetler, C. B., Morsi, D., Rucki, S., Broughton, S., Corrigan, B., Fitzgerald, J., Giuliano, K., Havener, P., \& Sheridan, E. A. (1998). Utilization-focused integrative reviews in a nursing service. Applied Nursing Research, 11(4), 195-206.

Triches, Eliane de Fátima; Aranda, M. A. de M. (2016). A formulação da Base Nacional Comum Curricular (BNCC) como ação da Política Educacional: breve levantamento bibliográfico (2014-2016). RealizAção - Revista on-line de Extensão e Cultura, 3(5). https://ojs.ufgd.edu.br/index.php/realizacao/article/view/6362

Turer, C. B., Brady, T. M., \& De Ferranti, S. D. (2018). Obesity, hypertension, and dyslipidemia in childhood are key modifiable antecedents of adult cardiovascular disease: A call to action. Circulation, 137(12), 1256-1259. https://doi.org/10.1161/CI RCULATIONAHA.118.032531

Viecheneski, Juliana Pinto; Carletto, M. (2012). Por que e para quê ensinar ciências para crianças. Simpósio Nacional De Ensino De Ciências Para Crianças, 1-12. http://www.sinect.com.br/2012/down.php?id=2729\&q=1

Whittemore, R. (2005). The integrative review: updated methodology. Journal of Advanced Nursing, 52(2), 546-553.

Young, M. (2007). Para que servem as escolas? Educação \& Sociedade, 28(101), 1287-1302. https://doi.org/10.1590/s0101-73302007000400002 
Research, Society and Development, v. 9, n. 11, e3759119865, 2020

(CC BY 4.0) | ISSN 2525-3409 | DOI: http://dx.doi.org/10.33448/rsd-v9i11.9865

Porcentagem de contribuição de cada autor no manuscrito

Susana Nogueira Diniz - 35\%

Thiago da Silva Lima $-20 \%$

Audrey de Souza Marques - 10\%

Cristina Eunice Okuyama - 35\% 